\title{
A case of maternally-inherited diabetes with deafness (MIDD)
}

\author{
Bakula M., Cavlovic Naglic M., Bakula M. ${ }^{*}$, Smircic Duvnjak L. \\ "Mekur", Clinical Hospital, "Vuk Vrhovac" University Clinic, Zagreb, Croatia \\ * "Sveti Duh" Clinical Hospital, Zagreb, Croatia
}

\section{INTRODUCTION}

MIDD is a rare form of monogenetic diabetes caused by a point mutation in human mitochondrial DNA at a position $3243 A>G^{1,2}$ (Figure 1). The syndrome is characterised by diabetes and sensoneural hearing impairment.

Additional clinical features include short stature, cardiomyopathy, myopathy, renal disease, macular distrophy and gastrointestinal disease $e^{3,4}$. The mutation is passed through maternal germline and all maternal relatives of an individual with MIDD are likely to be carriers of the mutation.

However, the clinical phenotype associated with the mutation may be very heterogeneous, even within the same family ${ }^{5}$

\section{CASE REPORT}

A lean 35-year old female patient (BMI 16) who was diagnosed with diabetes and mild hearing loss at the age of $\mathbf{2 9}$ was admited for blood glucose regulation.

Since childhood she has been suffering from migraine and at the age of twenty she was diagnosed with Wolff-Parkinson-White syndrome and prolapse of the mitral valve. After diagnosis of diabetes she was treated with metformin for four years, following basal insulin therapy and a year ago intensive insulin treatment was introduced. She tested negative for anti-islet humoral markers (ICA, anti-GAD and anti-IA2 antibodies) and her endogeneous insulin secretory reserve is partially maintained (C-peptide $0,46-0,75 \mathrm{nmol} / \mathrm{l})$. Bilateral neurosensory hearing loss was diagnosed and now required the use of hearing aid (Figure 2). The family history is positive for diabetes affecting her mother, uncle and maternal grandmother.

The clinical suspicion of MIDD was confirmed by the detection of mitochondrial DNA mutation m.3243A>G in patient's urinary epithelial cell DNA sample (Royal Devon and Exeter NHM Foundation Trust, UK). The patient's sister was advised to udergo genetic testing.

\section{DISCUSSION}

The patient we present was misdiagnosed for Type 2 diabetes in early course of disease and was treated with metformin for four years until intoduction of insulin therapy. The clinical suspicion of MIDD was raised six years after the onset of the disease upon the personal history of deafness, family history of diabetes and aditional features of the possible mitochondrial disorder: headaches, impairment of concentration, cardiac conduction defect and retinal pigment disgrupation.

The majority of MIDD patients usually require insulin therapy within two years and metformin is probably best avoided because of interference with mitochondrial function and increased risk od lactic acidosis.

\section{CONCLUSION}

It is assumed that MIDD affects up to $1 \%$ of all patients with diabetes but it often goes unrecognised and is misdiagnosed as either Type 1 or Type 2 diabetes.

Although it is not possible to predict the likely clinical course associated with this mutation due to variation in phenotype, it is important to suspect and confirm the diagnosis by the genetic testing in order to treat the patient optimally, to screen the patient for other clinical features of the syndrome and to search for the affected family members.

\section{REFERENCES}

1. Ballinger et al 1992, Nat Genet 1 , 11-15.

2. Maassen et al 1996, Exp Clin Endocrinol Diabetes 104 (3):205-11.

3. Murphy et al 2008, Diabet Med 25, 383-399.

4. Guillausseau et al 2001, Ann Intern Med 134: 721-728.

5. Nesbitt et al 2013, J Neurol Neurosurg Psychiatry 84, 936-938. 